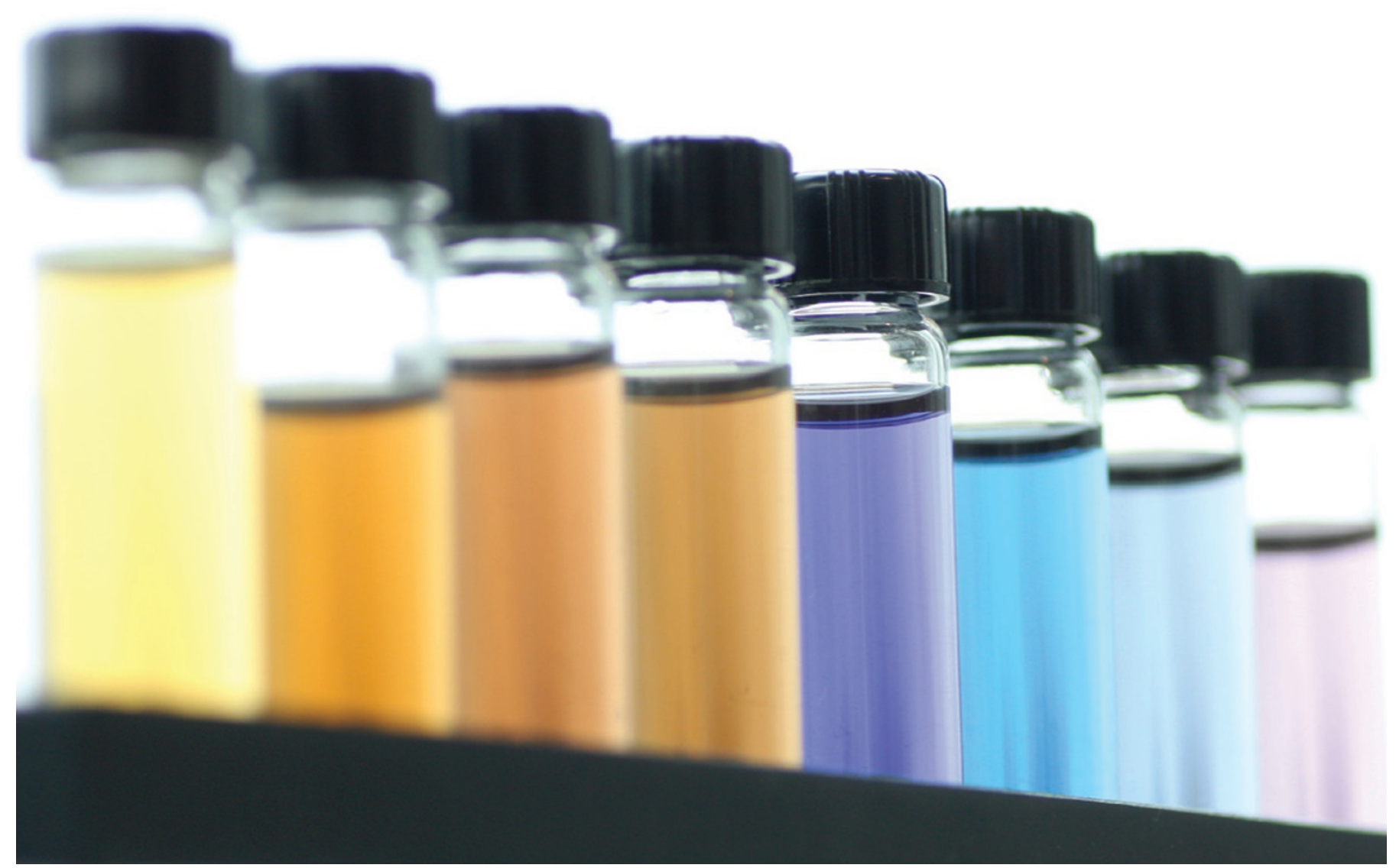

Their size and ability to absorb light are just two of the many qualities of gold nanoparticles that can be controlled — and these changes conveniently alter the colour of their solutions, making them ideal for use in diagnostics.

\title{
The new gold standard
}

\section{Prized for their versatility, optical properties and safety, gold nanoparticles are helping to image, diagnose and treat disease.}

\section{BY KAREN WEINTRAUB}

$\mathrm{I}$ $\mathrm{n}$ her lab in Cambridge, Massachusetts, Abigail Lytton-Jean holds up a burgundycoloured suspension of 15-nanometre gold particles. She pipettes a little into a vial, adds a bit of salt and shakes gently. Suddenly the red liquid turns deep blue. Gold particles carry a slight negative charge, so they repel one another. But the salt interferes with this repulsion and allows shorter-range attractive forces to take over; now, with the particles clumped together, the liquid appears blue. Researchers such as Lytton-Jean, a chemist at the Massachusetts Institute of Technology, can get any colour they like just by 'tuning' the size of the gold particles.

This colour control is one of the key assets of gold in a clinical setting. The size of gold particles, the charge, the hydrophobicity and the shape can all be manipulated to create nanoparticles that can home in on tumours, absorb light, deliver targeted drugs, and slip smoothly into a cell to silence genes.

"You can engineer them to behave nicely," says chemist Vincent Rotello of the University of Massachusetts Amherst, who works with gold nanoparticles. "You can stick a bunch of things on a gold nanoparticle and get it to have very controlled behaviour." It's easy, for example, to cover gold with sulphur atoms, and then you can attach medications, other metals, or almost anything a chemist might want, Rotello says.

Of the roughly 65,000 research papers on nanoparticles listed in PubMed since the first in 1978, more than 15\% mention gold, and most of those were in the past 5 years. "It's by far the most studied of these kinds of materials," says Paul Alivisatos, a nanotechnology researcher at the University of California, Berkeley, and director of the Lawrence
Berkeley National Laboratory. "Nothing else is even close."

The biomedical use of tiny bits of gold has taken off in the past 3-4 years, largely because the technology is so readily accessible, says Rotello. Fabrication of gold nanoparticles, it turns out, poses few difficulties even to those with limited laboratory experience - indeed, Rotello says, they're so easy to make that highschool students are doing it.

Most of the early biomedical work on nanoparticles (not just involving gold) was in cancer research, which still accounts for nearly $40 \%$ of nanotechnology submissions to the US Food and Drug Administration (FDA). Nanoparticles are particularly useful for targeted drug therapy because they move along the bloodstream but get trapped in the porous network of
DNATURE.COM

For some of the latest research on gold nanoparticles: go.nature.com/yL5las 
blood vessels that feed a tumour. Now, Rotello says, he and other chemists and bioengineers are branching out, using gold in a variety of applications ranging from detecting infection and gene mutations to experiments that send the nanoparticles across the blood-brain barrier to treat neurological conditions.

Biomedical work with gold nanoparticles can be categorized into four types of research: diagnosis, drug delivery, medical imaging and targeted killing of cells. Research is also beginning on so-called theranostic technologies, which combine diagnostics and treatment in a single procedure. Gold is an ideal theranostic because it can be used in so many different ways, says Dan Peer, a nanotechnologist who runs the nanomedicine lab at Tel Aviv University in Israel. In particular, its reflective nature can be exploited in diagnostics, and its ability to carry other compounds makes it an effective drug delivery platform.

And there's another intangible benefit to working with gold: people are intrigued by the metal, with its deep historical resonances relating to wealth and beauty. They've seen gold in stained glass windows, on the face of Egyptian mummies and in wedding rings. Nanotechnology itself can be traced back to Michael Faraday's early experiments with gold solutions. Gold research, Lytton-Jean says, "has benefited tremendously from its mystique".

\section{DIAGNOSTICS}

Conducting lab tests used to be the only way to diagnose many diseases and other health problems. A doctor would send a patient's blood sample to the hospital's lab and get results minutes, hours or even days later, by which time the patient's state may have changed. Diagnostics that can produce results quickly and be used in a range of settings, from the operating theatre or doctor's surgery to a rural clinic in Africa, are in high demand.

Taking diagnostics out of the lab requires the technology to be user friendly. Gold is a popular choice for diagnostics that need to be read easily, because it is biologically inert but still links easily to oligonucleotides (short, single strands of RNA or DNA), and it changes colour readily.

A good example of gold-based diagnostics is the FDA-approved Verigene detector. This device, manufactured by Nanosphere, based in Northbrook, Illinois, uses gold nanoparticles coated with oligonucleotides to capture and identify genetic sequences. It can detect a dozen bacteria known to cause infection. Typically, doctors prescribe broad-spectrum antibiotics when they suspect an infection and wait several days for lab results to identify the microorganism responsible. The Verigene detector can identify some infective agents within 2-3 hours, in time to prescribe more specific antibiotics and avoid unnecessary side effects, while limiting the use of broad-spectrum antibiotics that might lead to resistance.

\section{STEALTH FIGHTER}

Spherical nucleic acids can pass unharmed into cells to silence genes or deliver drugs.

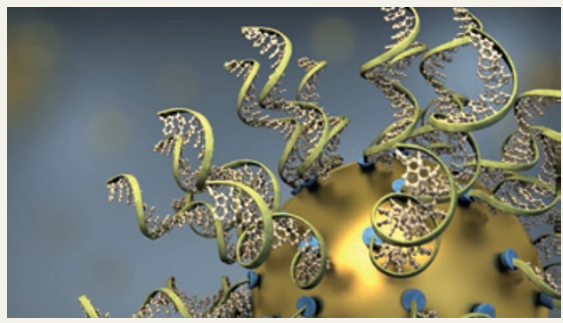

1. Spherical nucleic acids (SNAs) are made by decorating the outside of a gold nanoparticle with single-stranded or double-stranded nucleic acids.

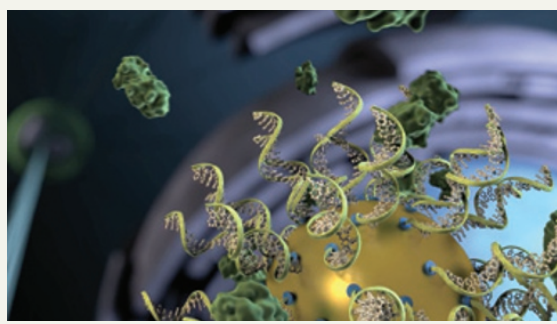

3. The spheres do not trigger an immune response and so are left intact inside the cell.

Verigene, based on the research of chemist Chad Mirkin at Northwestern University in Evanston, Illinois, was first approved in 2007 to test whether a patient is sensitive to the bloodthinning drug warfarin. The test is also used to detect the diarrhoea-causing Clostridium difficile bacteria and strains of influenza A.

Despite gold's reputation as a precious metal, the gold nanoparticles don't add much to the price of the medical device. Rotello says that even though $90 \%$ of his group's lab work involves gold, the metal accounts for only about $0.5 \%$ of its budget. According to Nanosphere's chief executive William Moffitt, roughly 35 million of its diagnostic tests can be manufactured from the amount of gold in one man's wedding ring.

\section{TINY TRANSPORTERS}

Because it is easy to attach things to gold, gold particles can act as a platform, carrying drugs inside tumours while also controlling their release.

The blood vessels that feed a tumour grow swiftly and are perforated with tiny holes. Nanoparticles that flow through most of the bloodstream get trapped in these pores and hence accumulate in tumours. This neatly sidesteps the problem of how to target treatment, a problem facing most cancer therapies.

The London-based pharmaceutical giant AstraZeneca, for example, recently announced a partnership with nanomedicine company CytImmune of Rockville, Maryland, to jointly develop a method for delivering cancer medication attached to a gold nanoparticle. CytImmune attaches molecules of tumour necrosis factor (TNF) to the surface of the gold

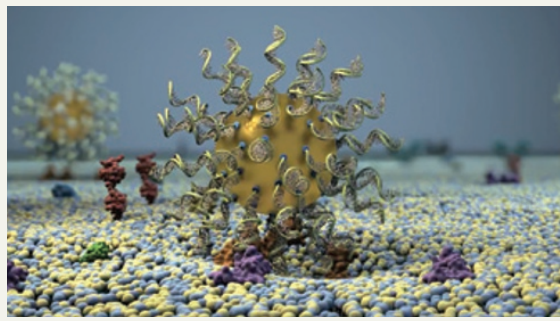

2. Cells readily take in SNAs, which therefore don't need to be packaged inside a virus, for example.

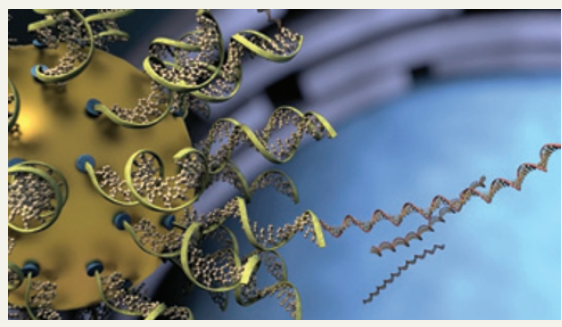

4. Nucleic acid strands on the sphere pair easily with their complements, allowing genes to be efficiently turned on or silenced.

particle. The TNF then binds preferentially to the endothelial cells in a tumour's blood vessels and kills them. Indeed, TNF was once a promising cancer treatment itself, but was dropped because it caused an inflammatory response when delivered systemically - a problem neatly overcome by using the gold nanoparticles. CytImmune has completed one phase 1 trial of its gold-plus-TNF drug, called CYT-6091, which showed good safety and tolerance. The company is now collaborating with AstraZeneca to add a chemotherapeutic drug to further improve the killing power, according to CytImmune's chief executive, Lawrence Tamarkin.

Tiny drug scaffolds can also be can used to arrange DNA or RNA into a framework called spherical nucleic acids (SNAs). When Mirkin built SNAs around gold particles and covered their surface with complementary antisense RNA or small interfering RNA, cells took them into the cytoplasm without eliciting an immune response.

The SNAs can easily pass through the skin's outer layer, so a suspension of them in a simple lotion could carry treatments for melanoma and other skin conditions directly to the hardto-reach target cells ${ }^{1}$. The SNAs can also cross the blood-brain barrier to target glioblastoma cells, Mirkin says. A company developed out of Mirkin's research, AuraSense Therapeutics, based in Skokie, Illinois, has preclinical data on the use of SNAs to treat glioblastoma, solid tumours and various skin diseases, and is working on the clinical development of all three.

Thanks to their ability to penetrate cells, SNAs can deliver oligonucleotides to regulate 
gene activity without the need for viruses or other potentially dangerous vectors, Mirkin says. In one recent study ${ }^{2}$, Mirkin chemically attached a monoclonal antibody that targets HER2, a growth factor that is implicated in breast cancer, to SNAs. Cells that express HER2 then take up the SNAs. Similar attachments involving a variety of antibodies, peptides, small molecules and other agents might improve the selectivity and potency of SNAs for a variety of gene-based diseases, the study concluded.

\section{HEALING WITH HEAT}

Another way to kill cancer cells is to use laser beams, but there are two main problems. One is the difficulty in pinpointing cancerous cells with a laser. The other is that the lethal beams can strike healthy cells. Gold nanoparticles might solve both problems.

Work by researchers including chemist and bioengineer Naomi Halas of Rice University in Houston, Texas, demonstrates that gold nanoparticles of various shapes can absorb nearinfrared light ${ }^{3}$. This range of the spectrum easily penetrates tissue without damaging healthy cells. The gold is made to congregate in the tumour, and when the light shines on these nanoparticles, it generates enough heat to kill the adjacent cell or to release drugs from carriers. "That gives you very high precision and very high efficacy" along with minimal side effects, Halas says.

Other work, from teams at the Georgia Institute of Technology in Atlanta and the University of California, San Francisco, has shown that gold nanoparticles optimized for their photothermal capabilities can also help scientists aim their lasers ${ }^{4}$. A company spun off from Halas's research, Nanospectra Biosciences in Houston, Texas, is conducting a pilot study of head and neck cancers that is due to end in mid-2013 (ref. 5). A trial testing the technology in lung cancer started in late 2012.

At the heart of Halas's technology are tiny glass shells coated in gold. These nanoshells are infused into the bloodstream a day before laser treatment. This gives the shells enough time to reach the tumour, but not long enough to be excreted. The nanoshells are coated in the compound polyethylene glycol, which shields the shells from the body's immune defence $^{6}$. Generally, gold particles of this size are excreted via the liver and kidneys.

The laser beam enters the body through a catheter; a few minutes of illumination ablates the cancer cells. The shells can also be imaged before they are zapped with the laser, helping to diagnose the disease, raising the possibility of using the technology as a theranostic.

These nanoshells might also serve as a delivery vector for gene silencing. Halas has recently shown that single-stranded antisense DNA oligonucleotides or double-stranded short-interfering RNA (siRNA) molecules can be carried on nanoshells and then released when they are exposed to ultraviolet light ${ }^{7}$.

\section{A SHARPER EYE}

Charles Craik, a pharmaceutical chemist at the University of California, San Francisco, explains his efforts to devise better ways to fight cancer. "When you look at a picture of a field of red poppies, if you look from a very high level, it's just a smear of red and you don't know what it is," he says. "When you go closer, you find it's a bunch of flowers." Go even closer, and it's clear that not all the poppies are red: a few are actually yellow.

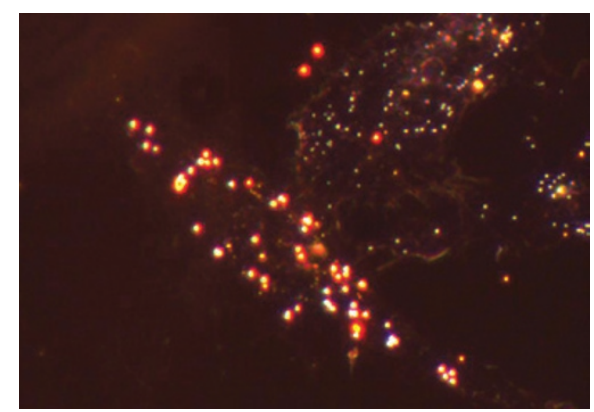

Golden glow: gold nanoparticles can enter cells for imaging or to deliver treatments.

To Craik, the yellow poppies represent the cancer stem cells in a tumour. If he can spot and count these yellow poppy stem cells, he can tell whether a cancer drug has killed them effectively - and therefore whether the cancer is likely to recur after treatment.

But as his metaphor suggests, it isn't easy to find them. Existing imaging technologies have limitations that make them impractical, he says. Green fluorescent proteins, for instance, emit light only briefly before they bleach out. Quantum dots - nanocrystals that fluoresce when exposed to light - can spot the cells, but the excitation of the crystal's electrons, which is what makes them visible, also makes them blink on and off, making it impossible to track single cells over time. The quantum dots have another serious drawback as well: they're toxic.

Gold solves both of these problems, Craik says. When light hits two neighbouring gold nanoparticles, the electrons on each are affected by the other, leading to an effect called plasmonic resonance. As the two particles are moved apart, this resonance "will affect the intensity of the light that's being reflected, and it will affect the colour", Craik explains.

One way researchers can exploit plasmonic resonance is by placing gold nanoparticles on either side of a peptide. When the peptide is intact, it appears one colour, Craik says. If the peptide is clipped - by an enzyme associated with cell death, for example - and the gold particles slip farther apart, their colour changes. Thanks to the plasmonic resonance, he says, "you can actually follow single-molecule cleavage."
This technique, which Craik developed in collaboration with Alivisatos, reveals which cells die during chemotherapy — and, he says, may eventually show whether stem cells are among them ${ }^{8}$. It also makes it easier to track the number of cells killed by various therapies, or the synergistic effects of a combination treatment versus a monotherapy, he says. Gold nanoparticles are "a beautiful tool for looking at synergistic drug therapy", Craik adds. "They're so exquisitely sensitive in terms of their ability to look at a single molecule."

\section{HOW SAFE?}

One lingering question about the biomedical use of gold is its safety. Gold is generally presumed to be safe, based on generations, if not centuries, of use. But Peer, of Tel Aviv University, warns against taking this for granted. "If it's only gold and no surface modification, there is no immune response," he says. In recent research, he and colleagues, including Rotello, found that modifying the gold surface with hydrophobic residues dictates the immune system's response ${ }^{9}$. In both in vitro and mouse studies, they found that an increase in hydrophobicity is correlated linearly with a rise in immune activation.

And Peer says that although gently activating the immune system could be a good thing in cancer care, it could be a disaster for treating neurodegenerative and inflammatory diseases. He thinks that all new applications of gold nanoparticles should be tested for liver toxicity, kidney toxicity and immune-system activation, and that the FDA should examine the possible hazards of nanoparticles more closely. "We all need to be more careful about how we define toxicity," he says.

But Halas says that research over the past few years has convinced her of the safety of gold nanoparticles in biomedical applications. "The picture of gold is getting clearer and clearer: it's boring. It's non-toxic," she says.

This safety, along with gold's versatility and the ease of manipulating it in the lab, suggests that researchers will continue to experiment with gold — and that it will remain the standard against which all other nanomaterials will be judged.

Karen Weintraub is a freelance science writer based in Cambridge, Massachusetts.

1. Zheng, D. D. et al. Proc. Natl Acad. Sci. USA 109, 11975-11980 (2012)

2. Zhang, K. et al. Am. Chem. Soc. 134, 16488-16491 (2012).

3. Huang, X. et al. Lasers Med. Sci. 23, 217-228 (2008).

4. Huang, X. et al. Am. Chem. Soc. 128, 2115-2120 (2006).

5. http://www.clinicaltrials.gov/ct2/show/ NCT01679470

6. Gad, S. C. et al. Int. J. Toxicol. 31, 584-594 (2012)

7. Huschka, R. et al. ACS Nano. 6, 7681-7691 (2012).

8. Jun, Y. W. et al. Proc. Natl Acad. Sci. USA 106, 17735-17740 (2009).

9. Moyano, D. F. et al. J. Am. Chem. Soc. $\mathbf{1 3 4}$ 3965-3967 (2012). 